Bull. Soc. math. France

130 (3), 2002, p. 387-408

\title{
INTERPOLATION SUR DES PERTURBATIONS D'ENSEMBLES PRODUITS
}

\author{
PAR DAMIEN ROY
}

À la mémoire de P. Robba

RÉSUmÉ. - On démontre un résultat concernant l'interpolation de fonctions analytiques sur une perturbation d'ensemble produit qui, dans le cas $p$-adique, répond à une conjecture de $\mathrm{P}$. Robba et, dans le cas complexe, complète des résultats antérieurs de E. Bombieri, S. Lang, D. Masser, J.-C. Moreau et M. Waldschmidt.

ABSTRACT (Interpolation on pertubations of cartesian products). - In 1970, E. Bombieri and S. Lang used analytic results of P. Lelong to establish a Schwarz lemma for a well distributed set of points in $\mathbb{C}^{n}$. Their result was extended to an interpolation lemma, first by D.W. Masser in the case of polynomials, then by M. Waldschmidt for analytic functions. J.-C. Moreau gave an analog of it over the real numbers and P. Robba in the $p$-adic realm. Robba also conjectured a $p$-adic interpolation lemma for the case where the set of points of interpolation is what he calls a perturbation of a product set, a situation which includes both the case of a well distributed set and the case of a cartesian product. In this paper, we present an algebraic proof of Robba's conjecture together with a generalization of it over the complex numbers.

Texte reçu le 30 mai 2001, accepté le 22 mars 2002

Damien Roy, Département de mathématiques, Université d'Ottawa, 585 King Edward, Ottawa, Ontario, Canada K1N 6N5

- E-mail : droy@uottawa.ca

Url : http://aix.uottawa.ca/ droy

Classification mathématique par sujets (2000). — primaire : 41A05, secondaire : 11J99, $32 \mathrm{E} 30$.

Mots clefs. - Interpolation, polynômes, lemme de Schwarz, fonctions analytiques, analyse $p$-adique, produits cartésiens, ensembles bien distribués.

Recherche partiellement subventionnée par le CRSNG et le CICMA.

BULletin DE LA SOCiÉtÉ MATHÉMATiQUE DE FRANCE 


\section{Introduction}

Le point de départ de ce travail est un très joli article de P. Robba [9] dans lequel il démontre des versions $p$-adiques de résultats d'interpolation sur un ensemble de points bien distribués. Ces résultats sont d'abord, à la source, un lemme de Schwarz de E. Bombieri et S. Lang pour les fonctions analytiques complexes [1], puis un lemme d'interpolation polynomiale de D.W. Masser (appendice II de [3]) ultérieurement raffiné par Masser lui-même (théorème A de [5]) et étendu aux fonctions analytiques par M. Waldschmidt (théorème 7.4.13 de [12]), et enfin un lemme d'interpolation polynomiale de J.-C. Moreau [6] qui répond à une question de Masser (appendice II de [3]) et que Moreau a étendu par la suite aux fonctions analytiques (proposition 2.6 de [7]).

Les résultats de Bombieri, Lang et Masser sont efficaces lorsque les points d'interpolation sont bien répartis dans une boule de $\mathbb{C}^{n}$ et leur démonstration s'appuie sur des propriétés de la masse moyenne des zéros d'une fonction analytique complexe. La méthode de Robba s'apparente à la leur car elle emploie une notion de multiplicité pondérée d'une fonction analytique $p$-adique aux propriétés analogues. Le résultat de Moreau quant à lui suppose que les points d'interpolation sont bien répartis dans une boule de $\mathbb{R}^{n}$ et sa démonstration est elle-aussi analytique. Le lecteur pourra consulter le chapitre 7 de [12] pour un aperçu plus complet des multiples travaux initiés par le résultat de Bombieri et Lang. Notons toutefois, comme me l'a signalé le rapporteur, que la première démonstration d'un lemme de Schwarz pour un ensemble bien distribué est antérieure et due à J.-P. Serre [11]. Elle est $p$-adique et repose sur une technique d'interpolation. Mentionnons aussi que les estimations de Robba pour la multiplicité pondérée (voir $\S$ III.2.7 de [9]) qui portent sur des germes d'hypersurfaces ont été étendus en codimension quelconque par J. Oesterlé [8].

Dans le même article, P. Robba démontre encore un lemme d'interpolation $p$-adique sur les produits cartésiens (théorème 3.1 de [9]), et il conjecture un résultat semblable pour la situation où l'ensemble des points d'interpolation est ce qu'il appelle une perturbation d'ensemble produit, une situation qui contient à la fois le cas d'un ensemble bien distribué et celui d'un ensemble produit (voir $\S 3.3$ de [9]). Nous nous proposons ici de démontrer cette séduisante idée de Robba à la fois sur un corps ultramétrique complet à valuation dense de caractéristique quelconque, et sur le corps des nombres complexes.

Notre argument ici est algébrique, grâce à l'application d'un critère de Waldschmidt-Moreau qui ramène le problème au cas des polynômes. Son principe ainsi que nos résultats principaux sont décrits au paragraphe suivant.

Remerciements. - Je remercie FRANÇOIS GRAMAIN pour ses commentaires sur une première version de ce travail. 


\section{Notations et résultats principaux}

On fixe dans toute la suite un entier positif $n$ et on désigne par $K$ le corps $\mathbb{C}$ des nombres complexes ou un corps valué ultramétrique complet dont le groupe de valuation $\left|K^{\times}\right|$est dense dans l'ouvert des réels positifs (comme par exemple le corps $p$-adique $\mathbb{C}_{p}$ pour un nombre premier $p$ ).

Pour un point $\alpha=\left(\alpha_{1}, \ldots, \alpha_{n}\right) \in K^{n}$ et un $n$-uplet $r=\left(r_{1}, \ldots, r_{n}\right)$ de nombres réels positifs, on note

$$
B(\alpha, r)=\left\{\left(x_{1}, \ldots, x_{n}\right) \in K^{n} ;\left|x_{i}-\alpha_{i}\right| \leq r_{i} \text { pour } i=1, \ldots, n\right\}
$$

le polydisque «fermé » de centre $\alpha$ et de multi-rayon $r$. On dit qu'une fonction $f: B(\alpha, r) \rightarrow K$ est analytique si elle est continue et représentée à l'intérieur de $B(\alpha, r)$ par une série de puissances convergente $\sum_{t \in \mathbb{N}^{n}} c_{t}(x-\alpha)^{t}$, avec la convention usuelle d'écriture

$$
(x-\alpha)^{t}=\prod_{i=1}^{n}\left(x_{i}-\alpha_{i}\right)^{t_{i}} \quad \text { pour } t=\left(t_{1}, \ldots, t_{n}\right) .
$$

Pour tout $t \in \mathbb{N}^{n}$, on désigne alors par $f^{[t]}(\alpha)$ le coefficient de $(x-\alpha)^{t}$ dans cette série, appelé dérivée divisée d'ordre $t$ de $f$ au point $\alpha$. Dans le cas où $\alpha=0$, on note $|f|_{r}$ la norme du supremum de $f$ sur $B(0, r)$. Rappelons que, si $K$ est ultramétrique et si $f$ est représentée par la série $\sum_{t \in \mathbb{N}^{n}} c_{t} x^{t}$ sur $B(0, r)$, alors on a $|f|_{r}=\max _{t \in \mathbb{N}^{n}}\left|c_{t}\right| r^{t}$. Pour $t=\left(t_{1}, \ldots, t_{n}\right) \in \mathbb{N}^{n}$, on emploie aussi les notations condensées

$$
t !=\left(t_{1}\right) ! \ldots\left(t_{n}\right) ! \quad \text { et } \quad|t|=t_{1}+\cdots+t_{n} .
$$

Si $u=\left(u_{1}, \ldots, u_{n}\right)$ est un autre élément de $\mathbb{N}^{n}$, on écrit $t<u$ (resp. $\left.t \leq u\right)$ pour signifier $t_{i}<u_{i}$ (resp. $\left.t_{i} \leq u_{i}\right)$ pour $i=1, \ldots, n$.

Pour tout sous-ensemble fini $\mathcal{E}$ de $K$ et tout point $a$ de $\mathcal{E}$, on définit

$$
\delta(a, \mathcal{E})=\min _{b \in \mathcal{E} \backslash a}|b-a| \text { et } \Delta(a, \mathcal{E})=\prod_{b \in \mathcal{E} \backslash a}|b-a|
$$

où $\mathcal{E} \backslash a$ est une notation abrégée pour $\mathcal{E} \backslash\{a\}$, l'ensemble $\mathcal{E}$ privé de $a$. On pose aussi

$$
\delta(\mathcal{E})=\min _{a \in \mathcal{E}} \delta(a, \mathcal{E}) \quad \text { et } \quad \Delta(\mathcal{E})=\min _{a \in \mathcal{E}} \Delta(a, \mathcal{E}) .
$$

On fixe enfin, dans toute la suite, des sous-ensembles finis $E_{1}, \ldots, E_{n}$ de $K$, de cardinalités respectives $S_{1}, \ldots, S_{n} \geq 2$ et un $n$-uplet d'entiers positifs $T=\left(T_{1}, \ldots, T_{n}\right)$. On pose

$$
E=E_{1} \times \cdots \times E_{n} \quad \text { et } \quad \mathcal{T}=\left\{t \in \mathbb{N}^{n} ; t<T\right\} .
$$

On pose aussi $L=\left(L_{1}, \ldots, L_{n}\right)$ avec $L_{i}=S_{i} T_{i}$ pour $i=1, \ldots, n$. On définit

$$
\delta(E)=\left(\delta\left(E_{1}\right), \ldots, \delta\left(E_{n}\right)\right) \quad \text { et } \quad \Delta(E)=\left(\Delta\left(E_{1}\right), \ldots, \Delta\left(E_{n}\right)\right)
$$

BULLETIN DE LA SOCiÉTÉ MATHÉMATIQUe DE FRANCE 
et, pour chaque $\alpha=\left(\alpha_{1}, \ldots, \alpha_{n}\right) \in E$, on pose

$$
\delta_{\alpha}=\left(\delta\left(\alpha_{1}, E_{1}\right), \ldots, \delta\left(\alpha_{n}, E_{n}\right)\right) .
$$

Comme ce sont des $n$-uplets de nombres réels positifs, les expressions $\delta(E)^{t}$, $\Delta(E)^{t}$ et $\delta_{\alpha}^{t}$ ont un sens pour tout $t \in \mathbb{Z}^{n}$.

Dans le cas ultramétrique, notre résultat principal est le suivant :

ThÉORÈme 1.1. - Supposons $K$ ultramétrique. Soit $\rho=\left(\rho_{1}, \ldots, \rho_{n}\right)$ un $n$-uplet de nombres réels positifs tel que $E \subset B(0, \rho)$, et soit $\Omega$ un sous-ensemble de $K^{n}$ tel que, pour tout $\alpha=\left(\alpha_{1}, \ldots, \alpha_{n}\right) \in E$, il existe $\omega=\left(\omega_{1}, \ldots, \omega_{n}\right) \in \Omega$ avec

$$
\left|\omega_{i}-\alpha_{i}\right|<\delta\left(\alpha_{i}, E_{i}\right) \quad \text { pour } i=1, \ldots, n .
$$

Alors, pour tout polynôme $P \in K[x]=K\left[x_{1}, \ldots, x_{n}\right]$ vérifiant $\operatorname{deg}_{x_{i}} P<L_{i}$ pour $i=1, \ldots, n$, on $a$

$$
|P|_{\rho} \leq \frac{\rho^{L}}{\Delta(E)^{T} \delta(E)^{T}} \max _{(\omega, t) \in \Omega \times \mathcal{T}}\left|P^{[t]}(\omega)\right| \delta(E)^{t} .
$$

En vertu de la définition des nombres $\delta\left(\alpha_{i}, E_{i}\right)$, un même point $\omega$ de $K^{n}$ ne peut vérifier la condition (1.1) pour plus d'un point $\alpha$ de $E$. Donc, dans l'énoncé du théorème 1.1, quitte à remplacer $\Omega$ par un sous-ensemble plus petit, on peut supposer que cette condition (1.1) détermine une bijection entre $E$ et $\Omega$. Dans ce cas, on dit que $\Omega$ est une perturbation de l'ensemble produit $E$. Cette notion généralise celle introduite par Robba au $§$ III.2.5.3 de [9]. Dans la définition de Robba, la condition (1.1) est remplacée par $\left|\omega_{i}-\alpha_{i}\right|<\delta$ pour $i=1, \ldots, n$, où $\delta=\min \delta\left(E_{i}\right)$.

Le corollaire suivant répond essentiellement à la conjecture formulée par Robba au $\S$ III.3.3 de [9].

Corollaire 1.2. - Sous les hypothèses du théorème 1.1, supposons que $\rho_{1}=\cdots=\rho_{n}$. Soient $r$ et $R$ des nombres réels positifs tels que $R \geq r \geq \rho_{1}$, soit $M$ un entier positif vérifiant $M \leq L_{i}$ pour $i=1, \ldots, n$ et soit $f: B(0,(R, \ldots, R)) \rightarrow K$ une fonction analytique. Alors, on a

$$
\begin{aligned}
|f|_{(r, \ldots, r)} \leq \max \left\{C_{1}\left(\frac{r}{\rho_{1}}\right)^{M} \max _{(\omega, t) \in \Omega \times \mathcal{T}}\left|f^{[t]}(\omega)\right| \delta(E)^{t}\right. & \\
& \left.C_{2}\left(\frac{r}{R}\right)^{M}|f|_{(R, \ldots, R)}\right\}
\end{aligned}
$$

avec $C_{1}=C_{2}=\rho_{1}^{|L|} /\left(\Delta(E)^{T} \delta(E)^{T}\right)$.

Dans l'énoncé de sa conjecture, Robba suppose en plus que le corps $K$ est de caractéristique 0 , que les facteurs $E_{1}, \ldots, E_{n}$ de $E$ ont même cardinalité $S_{1}=\cdots=S_{n}$, que $\Omega$ est une perturbation de $E$ au sens plus restreint rappelé plus haut, et qu'on a aussi $r=\rho_{1}, T_{1}=\cdots=T_{n}$ et $M=L_{1}=\cdots=L_{n}$.

TOME $130-2002-\mathrm{N}^{\mathrm{O}} 3$ 
Sous ces hypothèses, il propose que (1.2) est vrai avec $C_{1}=\left(\rho_{1} / \delta\right)^{M-1}$ et $C_{2}=1$, où $\delta=\min \delta\left(E_{i}\right)$. Cette valeur de $C_{1}$ se compare à celle du corollaire 1.2 puisque, sous les mêmes hypothèses, cette dernière est majorée par $\left(\rho_{1} / \delta\right)^{|L|}$. Par contre, la valeur conjecturée pour $C_{2}$ est incorrecte pour $n \geq 2$ et, comme on le verra au $\S 6$, on ne peut espérer améliorer nettement la constante $C_{2}$ du corollaire ni avec les hypothèses additionnelles de [9] indiquées ci-dessus, ni en se restreignant aux fonctions analytiques $f$ qui vérifient $f^{[t]}(\omega)=0$ pour tout $(\omega, t) \in \Omega \times \mathcal{T}$.

Le corollaire suivant généralise le théorème III.4.1 de [9].

Corollaire 1.3. - Supposons $K$ ultramétrique. On fixe un sous-corps localement compact $F$ de $K$. On note q la cardinalité de son corps résiduel, et $\lambda$ le générateur $>1$ de son groupe de valuation $\left|F^{\times}\right|$. Soient $\delta=\left(\delta_{1}, \ldots, \delta_{n}\right)$ et $\rho=\left(\rho_{1}, \ldots, \rho_{n}\right)$ des $n$-uplets d'éléments de $\left|F^{\times}\right|$avec $\delta<\rho$, et soit $\Omega$ un sousensemble de $K^{n}$ qui rencontre le polydisque $B(z, \delta)$ pour tout $z \in F^{n} \cap B(0, \rho)$. On pose $d=\log _{\lambda}(q), M_{i}=\left(\rho_{i} / \delta_{i}\right)^{d} T_{i}$ pour $i=1, \ldots, n$, et $M=M_{1}+\cdots+M_{n}$. Alors, pour tout polynôme $P \in K[x]$ vérifiant $\operatorname{deg}_{x_{i}} P<M_{i}$ pour $i=1, \ldots, n$, on a

$$
|P|_{\rho} \leq \lambda^{M /(q-1)} \max _{(\omega, t) \in \Omega \times \mathcal{T}}\left|P^{[t]}(\omega)\right| \delta^{t} .
$$

On verra au $\S 5$ comment une version ultramétrique du critère de Waldschmidt-Moreau permet de déduire le corollaire 1.2 du théorème 1.1. De la même façon, on trouve que le corollaire 1.3 implique les lemmes de Schwarz 4 et 5 de l'appendice 1 de [12] par D. Bertrand.

Dans le cas complexe, on démontre des résultats analogues :

THÉORÈme 1.4. - Supposons $K=\mathbb{C}$. Soit $\rho=\left(\rho_{1}, \ldots, \rho_{n}\right)$ un $n$-uplet de nombres réels positifs tel que $B\left(\alpha, 2 \delta_{\alpha}\right) \subseteq B(0, \rho)$ pour tout $\alpha \in E$. De plus, soient $m$ un entier positif et $\Omega$ un sous-ensemble de $\mathbb{C}^{n}$ vérifiant l'une ou l'autre des conditions suivantes :

(a) pour tout $\alpha \in E$, l'ensemble $\Omega$ rencontre $B\left(\alpha, \frac{1}{30} \delta(E)\right)$ et on a

$$
m \geq 4 n+\sum_{i=1}^{n} \frac{1}{T_{i}} \log _{2}(3 n)
$$

(b) pour tout $\alpha \in E$, l'ensemble $\Omega$ rencontre $B\left(\alpha, \frac{1}{15} \delta_{\alpha}\right)$ et on a

$$
m \geq 3 n+\sum_{i=1}^{n} \frac{1}{T_{i}} \log _{3}\left(3 n S_{i}\right)
$$

Alors, pour tout polynôme $P \in \mathbb{C}[x]=\mathbb{C}\left[x_{1}, \ldots, x_{n}\right]$ vérifiant $\operatorname{deg}_{x_{i}} P<L_{i} / m$ pour $i=1, \ldots, n$, on $a$

$$
|P|_{\rho} \leq 2^{m|T|} \frac{(8 \rho)^{L}}{\Delta(E)^{T} \delta(E)^{T}} \max _{(\omega, t) \in \Omega \times \mathcal{T}}\left|P^{[t]}(\omega)\right| \delta(E)^{t} .
$$

BULLETIN DE LA SOCIÉtÉ MATHÉMATIQUE DE FRANCE 
Le fait de supposer que le degré en $x_{i}$ du polynôme $P$ est strictement inférieur à $L_{i} / m$ au lieu de $L_{i}$ marque une différence essentielle entre le cas ultramétrique et le cas archimédien. C'est cette concession (qu'on retrouve aussi au lemme 3 de [6]) qui nous empêche d'étendre le théorème $\mathrm{B}$ de [5] à la situation d'un ensemble $\Omega$ comme ci-dessus. Néanmoins, en combinant le théorème $1.4 \mathrm{au}$ critère de Waldschmidt-Moreau, on obtient :

Corollaire 1.5. - Sous les hypothèses du théorème 1.4, supposons que $\rho_{1}=\cdots=\rho_{n}$. Soient $r$ et $R$ des nombres réels positifs tels que $R \geq r \geq \rho_{1}$, soit $M$ un entier positif ou nul vérifiant $M \leq L_{i} / m$ pour $i=1, \ldots, n$ et soit $f: B(0,(R, \ldots, R)) \rightarrow \mathbb{C}$ une fonction analytique. Alors, on a

$$
|f|_{(r, \ldots, r)} \leq C_{3}\left(\frac{r}{\rho_{1}}\right)^{M} \max _{(\omega, t) \in \Omega \times \mathcal{T}}\left|f^{[t]}(\omega)\right| \delta(E)^{t}+2 C_{3}\left(\frac{2^{n} r}{R}\right)^{M}|f|_{(R, \ldots, R)},
$$

avec $C_{3}=2^{m|T|}\left(8 \rho_{1}\right)^{|L|} /\left(\Delta(E)^{T} \delta(E)^{T}\right)$.

Pour les applications, la situation la plus intéressante demeure celle où l'ensemble $E$ est «bien distribué ». On trouve alors le résultat suivant qui, aux constantes près, contient le théorème 2.1 de [7] et généralise le corollaire $\mathrm{A}$ de [5] en y incluant des multiplicités.

Corollaire 1.6. - Soit $F$ un des corps $\mathbb{R}$ ou $\mathbb{C}$, soient $\delta=\left(\delta_{1}, \ldots, \delta_{n}\right)$ et $\rho=\left(\rho_{1}, \ldots, \rho_{n}\right)$ des $n$-uplets de nombres réels positifs avec $240 \delta<\rho$, et soit $\Omega$ un sous-ensemble de $\mathbb{C}^{n}$ qui rencontre le polydisque $B(z, \delta)$ pour tout $z \in F^{n} \cap B(0, \rho)$. On choisit un entier $m$ avec $m \geq n \log _{2}(48 n)$ et on pose $d=[F: \mathbb{R}], M_{i}=\left(\rho_{i} /\left(40 \delta_{i}\right)\right)^{d} T_{i}$ pour $i=1, \ldots, n$, et $M=M_{1}+\cdots+M_{n}$. Alors, pour tout polynôme $P \in \mathbb{C}[x]$ vérifiant $\operatorname{deg}_{x_{i}} P<M_{i} / m$ pour $1 \leq i \leq n$, on $a$

$$
|P|_{\rho} \leq 2^{m|T|+24 M} \max _{(\omega, t) \in \Omega \times \mathcal{T}}\left|P^{[t]}(\omega)\right|(40 \delta)^{t} .
$$

En combinant ce corollaire au critère de Waldschmidt-Moreau, on peut généraliser le lemme 7 de [4] au cas d'un réseau quelconque de $\mathbb{C}^{n}$, ce qui répond à une question de P. Grinspan au $\S 7$ de [2]. Notons en passant que les constantes qui apparaissent dans l'énoncé du corollaire pourraient être nettement améliorées grâce à un calcul plus soigné, adapté à cette situation.

L'idée de la preuve du théorème 1.1 est toute simple. Comme on l'a indiqué déjà, on peut d'abord supposer que $\Omega$ est une perturbation de $E$. On écrit le polynôme $P$ dans une base appropriée pour l'interpolation sur l'ensemble produit $E$. En évaluant cette expression et ses dérivées en les points de $\Omega$, on obtient un système d'équations linéaires en les coordonnées de $P$ dans cette base, avec autant d'équations que d'inconnues. Par bonheur, ce système est suffisamment proche d'une matrice diagonale pour qu'on puisse estimer sa solution, et par là estimer la norme de $P$.

TOME $130-2002-\mathrm{N}^{\mathrm{O}} 3$ 
En procédant de la même façon dans le cas complexe, on ne retrouverait pas les meilleurs résultats connus dans le cas d'un ensemble $E$ bien distribué, mais des résultats antérieurs comme le théorème A2 de [3]. C'est que la matrice du système qu'on obtient de cette manière n'est pas assez proche d'une matrice diagonale. Pour y remédier, on crée artificiellement une multiplicité additionnelle aux points de $\Omega$ en remplaçant $P$ par une puissance $P^{m}$ de $P$. Cela oblige à supposer que le degré de $P$ est plus petit peut-être qu'on ne l'aurait souhaité mais en contrepartie, cela permet de combiner un surplus d'équations pour produire un système mieux conditionné.

Le résultat dont nous aurons besoin concernant les systèmes d'équations linéaires est présenté au paragraphe suivant, ainsi qu'un autre lemme accessoire. Les preuves des théorèmes 1.1 et 1.4 et celles des corollaires sont données respectivement aux paragraphes 3,4 et 5 . On conclut au paragraphe 6 en établissant une borne inférieure pour la constante $C_{2}$ du corollaire 1.2 , et un raffinement de ce corollaire en l'absence de multiplicités.

\section{Résultats accessoires}

Les deux lemmes suivants se retrouvent dans différents contextes sous des formes variées. Il nous a tout de même paru utile d'en donner une preuve, faute de référence commode. Le premier fournit l'outil central de la démonstration des théorèmes 1.1 et 1.4 .

LEMME 2.1. - Soit $K$ un corps valué, soit I un ensemble fini, soient $c_{i, j}$, $((i, j) \in I \times I)$, des éléments de $K$, et $\rho_{i},(i \in I)$, des nombres réels positifs. On suppose que, pour tout $i \in I$, on a

$$
\left|c_{i, i}\right|=1 \quad \text { et }\left\{\begin{array}{l}
\max _{j \in I \backslash i}\left|c_{i, j}\right| \frac{\rho_{j}}{\rho_{i}}<1 \quad \text { si } K \text { est ultramétrique, } \\
\sum_{j \in I \backslash i}\left|c_{i, j}\right| \frac{\rho_{j}}{\rho_{i}} \leq \frac{1}{2} \quad \text { si } K \text { est archimédien. }
\end{array}\right.
$$

Alors, quel que soit $\left(b_{i}\right)_{i \in I} \in K^{I}$, le système d'équations linéaires

$$
\sum_{j \in I} c_{i, j} x_{j}=b_{i} \quad(i \in I)
$$

admet une et une seule solution $\left(x_{j}\right)_{j \in I} \in K^{I}$, et celle-ci vérifie

$$
\max _{j \in I} \frac{\left|x_{j}\right|}{\rho_{j}} \leq \gamma \max _{i \in I} \frac{\left|b_{i}\right|}{\rho_{i}}
$$

avec $\gamma=1$ si $K$ est ultramétrique et $\gamma=2$ si $K$ est archimédien. 
Démonstration. - Soit $\left(x_{j}\right)_{j \in I}$ un élément de $K^{I}$, et soit $\ell \in I$ un indice pour lequel le quotient $\left|x_{\ell}\right| / \rho_{\ell}$ est maximal. Si $K$ est ultramétrique, on a

$$
\max _{j \in I \backslash \ell}\left|c_{\ell, j} x_{j}\right| \leq \max _{j \in I \backslash \ell}\left|c_{\ell, j}\right| \rho_{j} \frac{\left|x_{\ell}\right|}{\rho_{\ell}} \leq\left|x_{\ell}\right|=\left|c_{\ell, \ell} x_{\ell}\right|,
$$

avec l'égalité partout si et seulement si $x_{\ell}=0$. Cela implique

$$
\left|\sum_{j \in I} c_{\ell, j} x_{j}\right|=\left|x_{\ell}\right| \geq \frac{\left|x_{\ell}\right|}{\gamma}
$$

Si $K$ est archimédien, cette dernière inégalité est encore vérifiée car

$$
\left|\sum_{j \in I} c_{\ell, j} x_{j}\right| \geq\left|c_{\ell, \ell} x_{\ell}\right|-\sum_{j \in I \backslash \ell}\left|c_{\ell, j} x_{j}\right| \geq\left|x_{\ell}\right|-\sum_{j \in I \backslash \ell}\left|c_{\ell, j}\right| \rho_{j} \frac{\left|x_{\ell}\right|}{\rho_{\ell}} \geq \frac{\left|x_{\ell}\right|}{2} .
$$

Donc, dans les deux cas, pour le choix de $b_{i}=0,(i \in I)$, le système $(2.1)$ n'admet que la solution triviale $x_{j}=0,(j \in I)$. On en déduit que la matrice de ce système est inversible et que, pour tout choix de $b_{i},(i \in I)$, il admet exactement une solution $x_{j},(j \in I)$. De plus, pour un choix d'indice $\ell$ comme ci-dessus, cette solution vérifie

$$
\max _{j \in I} \frac{\left|x_{j}\right|}{\rho_{j}}=\frac{\left|x_{\ell}\right|}{\rho_{\ell}} \leq \gamma \frac{\left|b_{\ell}\right|}{\rho_{\ell}} \leq \gamma \max _{i \in I} \frac{\left|b_{i}\right|}{\rho_{i}}
$$

Lemme 2.2. - Soit $\mathcal{E}$ un sous-ensemble fini de $\mathbb{C}$ de cardinalité $\geq 2$. Pour tout entier $s>2$ et tout $\alpha \in \mathcal{E}$, on a

$$
\sum_{\beta \in \mathcal{E} \backslash \alpha} \frac{1}{|\alpha-\beta|^{s}} \leq \frac{2}{s-2}\left(\frac{3}{\delta(\mathcal{E})}\right)^{s}
$$

Démonstration. - Fixons un choix d'entier $s>2$ et de $\alpha \in \mathcal{E}$, et posons $\rho=\frac{1}{2} \delta(\mathcal{E})$. Pour tout $\beta \in \mathcal{E}$ distinct de $\alpha$, et tout $z \in B(\beta, \rho)$, on a

$$
|z-\alpha| \leq|\alpha-\beta|+\rho \leq \frac{3}{2}|\alpha-\beta|
$$

On en déduit

$$
\int_{B(\beta, \rho)} \frac{1}{|z-\alpha|^{s}} \mathrm{~d} \mu \geq \pi \rho^{2}\left(\frac{2}{3|\alpha-\beta|}\right)^{s}
$$

où $\mu$ désigne la mesure de Lebesgue dans $\mathbb{C}$. Comme les intérieurs des boules $B(\beta, \rho)$ avec $\beta \in \mathcal{E}$ sont disjoints et contenus dans le complément $B(\alpha, \rho)^{c}$

TOME $130-2002-\mathrm{N}^{\mathrm{O}} 3$ 
de $B(\alpha, \rho)$ pour $\beta \neq \alpha$, on conclut

$$
\begin{aligned}
\sum_{\beta \in \mathcal{E} \backslash \alpha} \frac{1}{|\alpha-\beta|^{s}} & \leq \frac{1}{\pi \rho^{2}}\left(\frac{3}{2}\right)^{s} \sum_{\beta \in \mathcal{E} \backslash \alpha} \int_{B(\beta, \rho)} \frac{1}{|z-\alpha|^{s}} \mathrm{~d} \mu \\
& \leq \frac{1}{\pi \rho^{2}}\left(\frac{3}{2}\right)^{s} \int_{B(\alpha, \rho)^{c}} \frac{1}{|z-\alpha|^{s}} \mathrm{~d} \mu \\
& =\frac{2}{\rho^{2}}\left(\frac{3}{2}\right)^{s} \int_{\rho}^{\infty} \frac{r}{r^{s}} \mathrm{~d} r=\frac{2}{s-2}\left(\frac{3}{2 \rho}\right)^{s}
\end{aligned}
$$

\section{Preuve du théorème 1.1}

Avec les notations du paragraphe 1 , on pose, pour tout $\beta=\left(\beta_{1}, \ldots, \beta_{n}\right) \in E$,

$$
\varphi_{\beta}^{T}(x)=\prod_{i=1}^{n} \prod_{\gamma_{i} \in E_{i} \backslash \beta_{i}}\left(x_{i}-\gamma_{i}\right)^{T_{i}} .
$$

Alors les polynômes $(x-\beta)^{u} \varphi_{\beta}^{T}(x)$ avec $\beta \in E$ et $u \in \mathcal{T}$ forment une base du $K$ espace vectoriel $K[x]_{<L}$ constitué des polynômes $P \in K[x]$ avec $\operatorname{deg}_{x_{i}} P<L_{i}$ pour $i=1, \ldots, n$. Plus précisément, pour $P \in K[x]_{<L}$, on a

$$
P(x)=\sum_{(\beta, u) \in E \times \mathcal{T}} p_{(\beta, u)}(x-\beta)^{u} \varphi_{\beta}^{T}(x)
$$

où, pour chaque $(\beta, u) \in E \times \mathcal{T}$,

$$
p_{(\beta, u)}=\left(\frac{P}{\varphi_{\beta}^{T}}\right)^{[u]}(\beta) .
$$

Ces formules sont valables sur un corps $K$ quelconque.

Sous les hypothèses du théorème 1.1 , le corps $K$ est ultramétrique et, pour estimer les coefficients $p_{(\beta, u)}$ dans $(3.1)$, on procède comme suit. Pour chaque $\alpha \in E$, on choisit un élément $\omega$ de $\Omega$ qui vérifie la condition (1.1) du théorème, et on le note $\tilde{\alpha}$. Puis pour chaque $t \in \mathcal{T}$, on divise les deux membres de (3.1) $\operatorname{par} \varphi_{\alpha}^{T}(x)$, on calcule leurs dérivées divisées d'ordre $t$ et on les évalue au point $\tilde{\alpha}$. On obtient ainsi un système d'équations linéaires dont les équations et les inconnues sont indexées par $E \times \mathcal{T}$. Ce système s'écrit

$$
\sum_{(\beta, u) \in E \times \mathcal{T}} c_{(\alpha, t),(\beta, u)} p_{(\beta, u)}=b_{(\alpha, t)} \quad((\alpha, t) \in E \times \mathcal{T}),
$$

où

$$
c_{(\alpha, t),(\beta, u)}=\left((x-\beta)^{u} \frac{\varphi_{\beta}^{T}(x)}{\varphi_{\alpha}^{T}(x)}\right)_{x=\tilde{\alpha}}^{[t]} \text { et } \quad b_{(\alpha, t)}=\left(\frac{P}{\varphi_{\alpha}^{T}}\right)^{[t]}(\tilde{\alpha}) .
$$

Le premier des deux lemmes ci-dessous montre que ce système remplit les conditions du lemme 2.1 avec $I=E \times \mathcal{T}$ et $\rho_{(\alpha, t)}=\delta_{\alpha}^{T-t}$ pour tout $(\alpha, t) \in E \times \mathcal{T}$. Le second fournit une majoration des produits $\left|b_{(\alpha, t)}\right| \delta_{\alpha}^{t-T}$ qui interviennent 
dans la conclusion du lemme 2.1. Une fois ces résultats établis, un calcul succinct permet de compléter la démonstration du théorème.

Lemme 3.1. - Soient $(\alpha, t)$ et $(\beta, u)$ des éléments de $E \times \mathcal{T}$. On a

$$
\left|c_{(\alpha, t),(\beta, u)}\right| \frac{\delta_{\beta}^{T-u}}{\delta_{\alpha}^{T-t}} \leq 1
$$

avec égalité si et seulement si $(\alpha, t)=(\beta, u)$.

Démonstration. — On peut écrire

$$
c_{(\alpha, t),(\beta, u)}=g(\tilde{\alpha})
$$

où

$$
g(x)=\left((x-\beta)^{u} \frac{\varphi_{\beta}^{T}(x)}{\varphi_{\alpha}^{T}(x)}\right)^{[t]}=g_{1}\left(x_{1}\right) \cdots g_{n}\left(x_{n}\right)
$$

avec

$$
g_{i}\left(x_{i}\right)=\left(\frac{\left(x_{i}-\alpha_{i}\right)^{T_{i}}}{\left(x_{i}-\beta_{i}\right)^{T_{i}-u_{i}}}\right)^{\left[t_{i}\right]} \quad \text { pour } i=1, \ldots, n .
$$

Il suffit donc de montrer que, pour $i=1, \ldots, n$, on a

$$
\left|g_{i}\left(\tilde{\alpha}_{i}\right)\right| \leq \frac{\delta\left(\alpha_{i}, E_{i}\right)^{T_{i}-t_{i}}}{\delta\left(\beta_{i}, E_{i}\right)^{T_{i}-u_{i}}}
$$

avec égalité si et seulement si $\alpha_{i}=\beta_{i}$ et $t_{i}=u_{i}$. En d'autres termes, on est ramené à démontrer le lemme dans le cas où $n=1$, et on peut omettre partout les indices $i$.

Si $\alpha \neq \beta$, on a $|\tilde{\alpha}-\alpha|<|\alpha-\beta|$, donc $|\tilde{\alpha}-\beta|=|\alpha-\beta|$. Puisque

$$
g(x)=\sum_{k=0}^{t}\left(\begin{array}{l}
T \\
k
\end{array}\right)(x-\alpha)^{T-k}\left(\begin{array}{c}
T-u+t-k-1 \\
t-k
\end{array}\right) \frac{(-1)^{t-k}}{(x-\beta)^{T-u+t-k}},
$$

on obtient alors, comme requis,

$$
|g(\tilde{\alpha})| \leq \max _{0 \leq k \leq t} \frac{|\tilde{\alpha}-\alpha|^{T-k}}{|\alpha-\beta|^{T-u+t-k}} \leq \frac{|\tilde{\alpha}-\alpha|^{T-t}}{|\alpha-\beta|^{T-u}}<\frac{\delta(\alpha, E)^{T-t}}{\delta(\beta, E)^{T-u}}
$$

car $t<T$. Enfin, si $\alpha=\beta$, on a simplement $g(x)=\left((x-\alpha)^{u}\right)^{[t]}$. Lorsque $t<u$, on trouve de nouveau

$$
|g(\tilde{\alpha})| \leq|\tilde{\alpha}-\alpha|^{u-t}<\delta(\alpha, E)^{u-t}=\frac{\delta(\alpha, E)^{T-t}}{\delta(\alpha, E)^{T-u}} .
$$

La conclusion est la même lorsque $t>u$ car alors $g=0$. Enfin, si $t=u$, on a $g=1$, et le lemme est vérifié. 
LEMme 3.2. - Soit $(\alpha, t) \in E \times \mathcal{T}$. On a

$$
\left|b_{(\alpha, t)}\right| \delta_{\alpha}^{t-T} \leq \frac{1}{\Delta(E)^{T} \delta(E)^{T}} \max _{k \in \mathcal{T}}\left|P^{[k]}(\tilde{\alpha})\right| \delta(E)^{k} .
$$

Démonstration. - Soit $\delta=\left(\delta_{1}, \ldots, \delta_{n}\right)$ un $n$-uplet de nombres réels vérifiant $\left|\tilde{\alpha}_{i}-\alpha_{i}\right|<\delta_{i}<\delta\left(\alpha_{i}, E_{i}\right)$ pour $i=1, \ldots, n$. Pour tout $z \in B(\tilde{\alpha}, \delta)=B(\alpha, \delta)$, tout $i=1, \ldots, n$, et tout $\gamma_{i} \in E_{i} \backslash\left\{\alpha_{i}\right\}$, on a $\left|z_{i}-\gamma_{i}\right|=\left|\alpha_{i}-\gamma_{i}\right|$, donc $\left|\varphi_{\alpha}^{T}(z)\right|=\left|\varphi_{\alpha}^{T}(\alpha)\right|$. Alors, l'analogue ultramétrique des inégalités de Cauchy (ou un calcul direct) livre, pour tout $k \in \mathbb{N}^{n}$,

$$
\left|\left(\frac{1}{\varphi_{\alpha}^{T}}\right)^{[k]}(\tilde{\alpha})\right| \delta^{k} \leq \sup _{z \in B(\alpha, \delta)}\left|\frac{1}{\varphi_{\alpha}^{T}(z)}\right|=\frac{1}{\left|\varphi_{\alpha}^{T}(\alpha)\right|} .
$$

Comme $\delta$ peut être pris arbitrairement voisin de $\delta_{\alpha}$, on en déduit

$$
\left|b_{(\alpha, t)}\right| \delta_{\alpha}^{t}=\left|\sum_{0 \leq k \leq t} P^{[k]}(\tilde{\alpha})\left(\frac{1}{\varphi_{\alpha}^{T}}\right)^{[t-k]}(\tilde{\alpha})\right| \delta_{\alpha}^{t} \leq \frac{1}{\left|\varphi_{\alpha}^{T}(\alpha)\right|} \max _{0 \leq k \leq t}\left|P^{[k]}(\tilde{\alpha})\right| \delta_{\alpha}^{k},
$$

et la conclusion suit.

Preuve du théorème 1.1. - D'après le lemme 3.1, le système (3.2) vérifie les conditions d'application du lemme 2.1. Grâce au lemme 3.2, on en déduit

$$
\left|p_{(\beta, u)}\right| \rho^{u-T} \leq\left|p_{(\beta, u)}\right| \delta_{\beta}^{u-T} \leq \frac{1}{\Delta(E)^{T} \delta(E)^{T}} \max _{(\omega, t) \in \Omega \times \mathcal{T}}\left|P^{[t]}(\omega)\right| \delta(E)^{t}
$$

pour tout $(\beta, u) \in E \times \mathcal{T}$. Puisque la valeur absolue de $(x-\beta)^{u} \varphi_{\beta}^{T}(x)$ est majorée par $\rho^{u+L-T}$ sur $B(0, \rho)$, cela entraîne, comme requis

$$
|P|_{\rho} \leq \max _{(\beta, u) \in E \times \mathcal{T}}\left|p_{(\beta, u)}\right| \rho^{u+L-T} \leq \frac{\rho^{L}}{\Delta(E)^{T} \delta(E)^{T}} \max _{(\omega, t) \in \Omega \times \mathcal{T}}\left|P^{[t]}(\omega)\right| \delta(E)^{t} .
$$

\section{Preuve du théorème 1.4}

Soit $P \in \mathbb{C}[x]$ comme dans l'énoncé du théorème 1.4. Dans les notations du paragraphe 3 , on a $P^{m} \in \mathbb{C}[x]_{<L}$ et on peut écrire

$$
P^{m}(x)=\sum_{(\beta, u) \in E \times \mathcal{T}} p_{(\beta, u)}(x-\beta)^{u} \varphi_{\beta}^{T}(x)
$$

pour des nombres complexes $p_{(\beta, u)}$ dont il reste à majorer la valeur absolue en termes de la quantité

$$
A=\max _{(\omega, t) \in \Omega \times \mathcal{T}}\left|P^{[t]}(\omega)\right| \delta(E)^{t} .
$$

BULletin DE LA SOCIÉtÉ MATHÉMATIQUE DE FRANCE 
En vertu des hypothèses sur $m$, on peut trouver des entiers $m_{1}, \ldots, m_{n}$ qui vérifient $m_{1}+\cdots+m_{n}=m+n-1$ et, pour $i=1, \ldots, n$,

$$
m_{i} \geq \begin{cases}4+\frac{1}{T_{i}} \log _{2}(3 n) & \text { dans le cas }(\mathrm{a}), \\ 3+\frac{1}{T_{i}} \log _{3}\left(3 n S_{i}\right) & \text { dans le cas }(\mathrm{b}) .\end{cases}
$$

On pose alors

$$
U=\left(U_{1}, \ldots, U_{n}\right), \quad \text { avec } \quad U_{i}=m_{i} T_{i} \quad \text { pour } i=1, \ldots, n .
$$

Selon le cas (a) ou (b), on peut aussi choisir, pour chaque $\alpha \in E$, un élément $\tilde{\alpha}$ de $\Omega$ dans le polydisque correspondant $B\left(\alpha, \frac{1}{30} \delta(E)\right)$ ou $B\left(\alpha, \frac{1}{15} \delta_{\alpha}\right)$. Notre approche est basée sur le fait que les valeurs absolues des dérivées divisées de $P^{m}$ d'ordre $<U$ aux points $\tilde{\alpha}$ peuvent être majorées en termes de $A$ et de $|P|_{\rho}$ (voir la preuve du lemme 4.3).

Pour un couple $(\alpha, t) \in E \times \mathcal{T}$ fixé, (4.1) implique

$$
\left(\frac{P^{m}(x)}{\varphi_{\alpha}^{T}(x)}\right)^{[t]}=\sum_{(\beta, u) \in E \times \mathcal{T}} p_{(\beta, u)}\left((x-\beta)^{u} \frac{\varphi_{\beta}^{T}(x)}{\varphi_{\alpha}^{T}(x)}\right)^{[t]} .
$$

En tronquant à l'ordre $U-t$ les développements de Taylor des deux membres de cette égalité autour du point $\tilde{\alpha}$, et en évaluant au point $\alpha$ les développements tronqués, on obtient une équation linéaire en les $p_{(\beta, u)}$. On est ainsi conduit à un système d'équations linéaires

$$
\sum_{(\beta, u) \in E \times \mathcal{T}} c_{(\alpha, t),(\beta, u)} p_{(\beta, u)}=b_{(\alpha, t)} \quad((\alpha, t) \in E \times \mathcal{T}),
$$

où

$$
c_{(\alpha, t),(\beta, u)}=\sum_{0 \leq k<U-t}\left((x-\beta)^{u} \frac{\varphi_{\beta}^{T}(x)}{\varphi_{\alpha}^{T}(x)}\right)_{x=\tilde{\alpha}}^{[t][k]}(\alpha-\tilde{\alpha})^{k}
$$

et

$$
b_{(\alpha, t)}=\sum_{0 \leq k<U-t}\left(\frac{P^{m}(x)}{\varphi_{\alpha}^{T}(x)}\right)_{x=\tilde{\alpha}}^{[t][k]}(\alpha-\tilde{\alpha})^{k} .
$$

Dans ces formules, l'exposant $[t][k]$ indique qu'on prend successivement la dérivée divisée d'ordre $t$ de la fonction, puis la dérivée divisée d'ordre $k$ du résultat; l'indice $x=\tilde{\alpha}$ signifie qu'on évalue le tout au point $x=\tilde{\alpha}$. Partant de là, les calculs sont semblables à ceux du paragraphe 3 , quoique plus élaborés. On établit d'abord trois lemmes.

Lemme 4.1. - Soient $(\alpha, t)$ et $(\beta, u)$ des éléments de $E \times \mathcal{T}$. L'expression

$$
\left|c_{(\alpha, t),(\beta, u)}\right| \frac{\delta_{\beta}^{T-u}}{\delta_{\alpha}^{T-t}}
$$

TOME $130-2002-\mathrm{N}^{\mathrm{O}} 3$ 
vaut 0 s'il existe un indice $i$ tel que $\alpha_{i}=\beta_{i}$ et $t_{i} \neq u_{i}$. Sinon, elle est majorée par

$$
\prod_{\alpha_{i} \neq \beta_{i}}\left(\frac{5^{U_{i}}}{2^{u_{i}+1}}\left|\frac{\alpha_{i}-\tilde{\alpha}_{i}}{\alpha_{i}-\beta_{i}}\right|^{U_{i}-T_{i}}\right)
$$

où le produit porte sur tous les indices $i$ tels que $\alpha_{i} \neq \beta_{i}$. Enfin, cette expression est égale à 1 si $(\alpha, t)=(\beta, u)$.

Démonstration. - En reprenant les notations (3.3) et (3.4) introduites dans la preuve du lemme 3.1, on trouve

$$
\begin{aligned}
\left|c_{(\alpha, t),(\beta, u)}\right| \frac{\delta_{\beta}^{T-u}}{\delta_{\alpha}^{T-t}} & =\left|\sum_{0 \leq k<U-t} g^{[k]}(\tilde{\alpha})(\alpha-\tilde{\alpha})^{k}\right| \frac{\delta_{\beta}^{T-u}}{\delta_{\alpha}^{T-t}} \\
& =\left|\sum_{0 \leq k<U-t} \prod_{i=1}^{n}\left(g_{i}^{\left[k_{i}\right]}\left(\tilde{\alpha}_{i}\right)\left(\alpha_{i}-\tilde{\alpha}_{i}\right)^{k_{i}}\right)\right| \frac{\delta_{\beta}^{T-u}}{\delta_{\alpha}^{T-t}} \\
& =\prod_{i=1}^{n}\left(\left|\sum_{k_{i}=0}^{U_{i}-t_{i}-1} g_{i}^{\left[k_{i}\right]}\left(\tilde{\alpha}_{i}\right)\left(\alpha_{i}-\tilde{\alpha}_{i}\right)^{k_{i}}\right| \frac{\delta\left(\beta_{i}, E_{i}\right)^{T_{i}-u_{i}}}{\delta\left(\alpha_{i}, E_{i}\right)^{T_{i}-t_{i}}}\right) .
\end{aligned}
$$

Il suffit donc de démontrer le lemme dans le cas $n=1$, hypothèse sous laquelle on peut omettre partout les indices $i$.

La formule intégrale du reste dans le développement de Taylor montre qu'il existe un point $\theta$ sur le segment de droite qui joint $\alpha$ à $\tilde{\alpha}$ dans $\mathbb{C}$ tel que

$$
\left|g(\alpha)-\sum_{k=0}^{U-t-1} g^{[k]}(\tilde{\alpha})(\alpha-\tilde{\alpha})^{k}\right| \leq\left|g^{[U-t]}(\theta)\right| \cdot|\alpha-\tilde{\alpha}|^{U-t} .
$$

Si $\alpha=\beta$, on a $g(x)=\left((x-\alpha)^{u}\right)^{[t]}$. Comme $u<U$, on en déduit $g^{[U-t]}=0$ et par suite

$$
\sum_{k=0}^{U-t-1} g^{[k]}(\tilde{\alpha})(\alpha-\tilde{\alpha})^{k}=g(\alpha)= \begin{cases}1 & \text { si } t=u, \\ 0 & \text { si } t \neq u .\end{cases}
$$

Donc le lemme est démontré dans ce cas.

Supposons maintenant $\alpha \neq \beta$. On trouve $g(\alpha)=0$ et la relation (4.3) livre

$$
\begin{aligned}
\left|\sum_{k=0}^{U-t-1} g^{[k]}(\tilde{\alpha})(\alpha-\tilde{\alpha})^{k}\right| & \frac{\delta(\beta, E)^{T-u}}{\delta(\alpha, E)^{T-t}} \\
& \leq\left|g^{[U-t]}(\theta)\right| \cdot|\alpha-\tilde{\alpha}|^{U-t} \frac{\delta(\beta, E)^{T-u}}{\delta(\alpha, E)^{T-t}} \\
& \leq\left|g^{[U-t]}(\theta)\right| \cdot|\alpha-\tilde{\alpha}|^{U-T} \cdot|\alpha-\beta|^{T-u}
\end{aligned}
$$

BULLETIN DE LA SOCIÉTÉ MATHÉMATIQUE DE FRANCE 
en utilisant $|\alpha-\tilde{\alpha}| \leq \delta(\alpha, E)$ et $\delta(\beta, E) \leq|\alpha-\beta|$. D'autre part, on a

$$
\begin{aligned}
& g^{[U-t]}(x)=\left(\begin{array}{c}
U \\
t
\end{array}\right)\left(\frac{(x-\alpha)^{T}}{(x-\beta)^{T-u}}\right)^{[U]} \\
& =\left(\begin{array}{c}
U \\
t
\end{array}\right) \sum_{k=0}^{T}\left(\begin{array}{l}
T \\
k
\end{array}\right)(x-\alpha)^{T-k}\left(\begin{array}{c}
T-u+U-k-1 \\
U-k
\end{array}\right) \frac{(-1)^{U-k}}{(x-\beta)^{T-u+U-k}} .
\end{aligned}
$$

Comme $|\theta-\alpha| \leq|\alpha-\tilde{\alpha}| \leq \frac{1}{15}|\alpha-\beta|$, on a $|\theta-\beta| \geq|\alpha-\beta|-|\theta-\alpha| \geq \frac{14}{15}|\alpha-\beta|$, et on en déduit

$$
\begin{aligned}
\left|g^{[U-t]}(\theta)\right| & \leq 2^{U} \sum_{k=0}^{T}\left(\begin{array}{l}
T \\
k
\end{array}\right)\left(\frac{|\alpha-\beta|}{15}\right)^{T-k} 2^{T-u+U-k-1}\left(\frac{15}{14|\alpha-\beta|}\right)^{T-u+U-k} \\
& =2^{2 U-u-1}\left(\frac{15}{14}\right)^{U-u}\left(\frac{8}{7}\right)^{T}|\alpha-\beta|^{u-U} \leq \frac{5^{U}}{2^{u+1}}|\alpha-\beta|^{u-U} .
\end{aligned}
$$

En combinant cette inégalité avec (4.4), on obtient la majoration requise.

Lemme 4.2. - Soit $(\alpha, t) \in E \times \mathcal{T}$. On a

$$
\sum_{(\beta, u) \neq(\alpha, t)}\left|c_{(\alpha, t),(\beta, u)}\right| \frac{\delta_{\beta}^{T-u}}{\delta_{\alpha}^{T-t}} \leq \frac{1}{2}
$$

où la somme porte sur tous les couples $(\beta, u) \in E \times \mathcal{T}$ distincts de $(\alpha, t)$.

Démonstration. - Grâce au lemme 4.1, on a

$$
\begin{aligned}
& 1+\sum_{(\beta, u) \neq(\alpha, t)}\left|c_{(\alpha, t),(\beta, u)}\right| \frac{\delta_{\beta}^{T-u}}{\delta_{\alpha}^{T-t}}=\sum_{(\beta, u) \in E \times \mathcal{T}}\left|c_{(\alpha, t),(\beta, u)}\right| \frac{\delta_{\beta}^{T-u}}{\delta_{\alpha}^{T-t}} \\
& \leq \prod_{i=1}^{n}\left(1+\sum_{\beta_{i} \in E_{i} \backslash \alpha_{i}} \sum_{u_{i}=0}^{T_{i}-1} \frac{5^{U_{i}}}{2^{u_{i}+1}}\left|\frac{\alpha_{i}-\tilde{\alpha}_{i}}{\alpha_{i}-\beta_{i}}\right|^{U_{i}-T_{i}}\right) \\
& \leq \prod_{i=1}^{n}\left(1+\sum_{\beta_{i} \in E_{i} \backslash \alpha_{i}} 5^{U_{i}}\left|\frac{\alpha_{i}-\tilde{\alpha}_{i}}{\alpha_{i}-\beta_{i}}\right|^{U_{i}-T_{i}}\right) .
\end{aligned}
$$

Comme $(1+1 /(3 n))^{n} \leq \frac{3}{2}$, il suffit, pour conclure, de montrer que

$$
\sum_{\beta_{i} \in E_{i} \backslash \alpha_{i}} 5^{U_{i}}\left|\frac{\alpha_{i}-\tilde{\alpha}_{i}}{\alpha_{i}-\beta_{i}}\right|^{U_{i}-T_{i}} \leq \frac{1}{3 n} \quad \text { pour } i=1, \ldots, n .
$$

Fixons donc un indice $i$. Dans le cas (a), on a $m_{i} \geq 5$, donc $U_{i}-T_{i}=$ $\left(m_{i}-1\right) T_{i} \geq 4$ et, grâce au lemme 2.2 , on obtient bien

$$
\sum_{\beta_{i} \in E_{i} \backslash \alpha_{i}} 5^{U_{i}}\left|\frac{\alpha_{i}-\tilde{\alpha}_{i}}{\alpha_{i}-\beta_{i}}\right|^{U_{i}-T_{i}} \leq 5^{U_{i}}\left(\frac{3\left|\alpha_{i}-\tilde{\alpha}_{i}\right|}{\delta\left(E_{i}\right)}\right)^{U_{i}-T_{i}} \leq 5^{T_{i}}\left(\frac{1}{2}\right)^{U_{i}-T_{i}} \leq \frac{1}{3 n} .
$$

TOME $130-2002-\mathrm{N}^{\mathrm{O}} 3$ 
Dans le cas (b), la conclusion est la même :

$$
\sum_{\beta_{i} \in E_{i} \backslash \alpha_{i}} 5^{U_{i}}\left|\frac{\alpha_{i}-\tilde{\alpha}_{i}}{\alpha_{i}-\beta_{i}}\right|^{U_{i}-T_{i}} \leq \sum_{\beta_{i} \in E_{i} \backslash \alpha_{i}} 5^{U_{i}}\left(\frac{1}{15}\right)^{U_{i}-T_{i}} \leq S_{i}\left(\frac{1}{3}\right)^{\left(m_{i}-3\right) T_{i}} \leq \frac{1}{3 n} .
$$

LEMmE 4.3. - Soit $(\alpha, t) \in E \times \mathcal{T}$. On a

$$
\left|b_{(\alpha, t)}\right| \delta_{\alpha}^{t-T} \leq 2^{|L|+2|T|+n(m-2)}|P|_{\rho}^{m-1} \frac{A}{\delta(E)^{T} \Delta(E)^{T}}
$$

Démonstration. - On procède en quatre étapes.

(i) Comme $B\left(\tilde{\alpha}, \delta_{\alpha}\right) \subseteq B\left(\alpha, 2 \delta_{\alpha}\right) \subseteq B(0, \rho)$, les inégalités de Cauchy donnent

$$
\left|P^{[u]}(\tilde{\alpha})\right| \delta_{\alpha}^{u} \leq|P|_{\rho}
$$

pour tout $u \in \mathbb{N}^{n}$.

(ii) Pour tout $k \in \mathbb{N}^{n}$ avec $k<U$, on a

$$
\left|\left(P^{m}\right)^{[k]}(\tilde{\alpha})\right| \delta_{\alpha}^{k-T} \leq 2^{|k|+n(m-1)}|P|_{\rho}^{m-1} \frac{A}{\delta(E)^{T}} .
$$

En effet, on a $\left(P^{m}\right)^{[k]}=\sum P^{\left[k^{(1)}\right]} \cdots P^{\left[k^{(m)}\right]}$ où la somme porte sur toutes les décompositions de $k$ en somme de $m$ éléments $k^{(1)}, \ldots, k^{(m)}$ de $\mathbb{N}^{n}$. Pour une décomposition donnée et pour un indice $i$ fixé, on a

$$
k_{i}=\sum_{j=1}^{m} k_{i}^{(j)}<U_{i}=m_{i} T_{i}
$$

donc $k_{i}^{(j)} \geq T_{i}$ pour au plus $m_{i}-1$ indices $j$. Par suite, on a $k^{(j)} \notin \mathcal{T}$ pour au plus $\sum\left(m_{i}-1\right)=m-1$ indices $j$. On en déduit, grâce à (4.5),

$$
\left|P^{\left[k^{(1)}\right]}(\tilde{\alpha}) \cdots P^{\left[k^{(m)}\right]}(\tilde{\alpha})\right| \delta_{\alpha}^{k}=\prod_{j=1}^{m}\left(\left|P^{\left[k^{(j)}\right]}(\tilde{\alpha})\right| \delta_{\alpha}^{k^{(j)}}\right) \leq|P|_{\rho}^{m-1} \max _{t \in \mathcal{T}}\left|P^{[t]}(\tilde{\alpha})\right| \delta_{\alpha}^{t} .
$$

L'inégalité (4.6) s'ensuit, puisque $\delta_{\alpha} \geq \delta(E)$ et que le nombre de décompositions de $k$ est

$$
\prod_{i=1}^{n}\left(\begin{array}{c}
k_{i}+m-1 \\
m-1
\end{array}\right) \leq \prod_{i=1}^{n} 2^{k_{i}+m-1}=2^{|k|+n(m-1)} .
$$

(iii) Puisque $B\left(\tilde{\alpha}, \frac{1}{4} \delta_{\alpha}\right) \subseteq B\left(\alpha, \frac{1}{2} \delta_{\alpha}\right)$, les inégalités de Cauchy livrent encore

$$
\left|\left(\frac{1}{\varphi_{\alpha}^{T}}\right)^{[k]}(\tilde{\alpha})\right|\left(\frac{\delta_{\alpha}}{4}\right)^{k} \leq \sup \left\{\left|\frac{1}{\varphi_{\alpha}^{T}(z)}\right| ; z \in B\left(\alpha, \frac{\delta_{\alpha}}{2}\right)\right\} \leq \frac{2^{|L|-|T|}}{\Delta(E)^{T}}
$$

BULletin DE LA SOCiÉtÉ MathÉmatique DE FRANCE 
car $\varphi_{\alpha}^{T}(z)$ est un produit de $|L|-|T|$ facteurs de la forme $\left|z_{i}-\beta_{i}\right|^{T_{i}}$ avec $\beta_{i} \in E_{i} \backslash\left\{\alpha_{i}\right\}$ et, pour un tel $\beta_{i}$, on a

$$
\left|z_{i}-\beta_{i}\right| \geq\left|\alpha_{i}-\beta_{i}\right|-\left|z_{i}-\alpha_{i}\right| \geq \frac{1}{2}\left|\alpha_{i}-\beta_{i}\right|
$$

lorsque $\left|z_{i}-\alpha_{i}\right| \leq \frac{1}{2} \delta\left(\alpha_{i}, E_{i}\right)$.

(iv) Pour tout $u \in \mathbb{N}^{n}$ avec $u<U$, on trouve, grâce à (4.6) et (4.7),

$$
\begin{aligned}
\left|\left(\frac{P^{m}}{\varphi_{\alpha}^{T}}\right)^{[u]}(\tilde{\alpha})\right| \delta_{\alpha}^{u-T} & =\left|\sum_{k \leq u}\left(P^{m}\right)^{[k]}(\tilde{\alpha})\left(\frac{1}{\varphi_{\alpha}^{T}}\right)^{[u-k]}(\tilde{\alpha})\right| \delta_{\alpha}^{u-T} \\
& \leq \sum_{k \leq u} 2^{|k|+n(m-1)}|P|_{\rho}^{m-1} \frac{A}{\delta(E)^{T}} \frac{2^{|L|-|T|}}{\Delta(E)^{T}} 4^{|u|-|k|} \\
& \leq 2^{|L|+2|u|-|T|+n m}|P|_{\rho}^{m-1} \frac{A}{\Delta(E)^{T} \delta(E)^{T}}
\end{aligned}
$$

car $\sum_{k \leq u} 2^{-|k|} \leq 2^{n}$. On en déduit

$$
\begin{aligned}
\left|b_{(\alpha, t)}\right| \delta_{\alpha}^{t-T} & =\left|\sum_{k<U-t}\left(\frac{P^{m}}{\varphi_{\alpha}^{T}}\right)^{[t][k]}(\tilde{\alpha})(\alpha-\tilde{\alpha})^{k}\right| \delta_{\alpha}^{t-T} \\
& \leq \sum_{k<U-t} \frac{(t+k) !}{t ! k !}\left|\left(\frac{P^{m}}{\varphi_{\alpha}^{T}}\right)^{[t+k]}(\tilde{\alpha})\right|\left(\frac{\delta_{\alpha}}{8}\right)^{k} \delta_{\alpha}^{t-T} \\
& \leq 2^{|L|+2|t|-|T|+n m}|P|_{\rho}^{m-1} \frac{A}{\Delta(E)^{T} \delta(E)^{T}} \sum_{k \in \mathbb{N}^{n}} \frac{(t+k) !}{t ! k !} 2^{-|k|} \\
& =2^{|L|+3|t|-|T|+n(m+1)}|P|_{\rho}^{m-1} \frac{A}{\Delta(E)^{T} \delta(E)^{T}} .
\end{aligned}
$$

La conclusion du lemme s'ensuit puisque $|t| \leq|T|-n$.

Preuve du théorème 1.4. - D'après le lemme 4.2, le système (4.2) vérifie les conditions d'application du lemme 2.1. Grâce au lemme 4.3, on en déduit

$$
\left|p_{(\beta, u)}\right| \rho^{u-T} \leq\left|p_{(\beta, u)}\right| \delta_{\beta}^{u-T} \leq 2^{1+|L|+2|T|+n(m-2)}|P|_{\rho}^{m-1} \frac{A}{\Delta(E)^{T} \delta(E)^{T}}
$$

pour tout $(\beta, u) \in E \times \mathcal{T}$. Comme la valeur absolue de $(x-\beta)^{u} \varphi_{\beta}^{T}(x)$ est majorée par $(2 \rho)^{u+L-T}$ sur $B(0, \rho)$, cela entraîne

$$
\begin{aligned}
|P|_{\rho}^{m}=\left|P^{m}\right|_{\rho} & \leq \sum_{(\beta, u) \in E \times \mathcal{T}}\left|p_{(\beta, u)}\right|(2 \rho)^{u+L-T} \\
& \leq 2^{|L|-n} \max _{(\beta, u) \in E \times \mathcal{T}}\left|p_{(\beta, u)}\right|(2 \rho)^{u+L-T} \\
& \leq 2^{1+2|T|+n(m-4)}|P|_{\rho}^{m-1} \frac{(8 \rho)^{L} A}{\Delta(E)^{T} \delta(E)^{T}} .
\end{aligned}
$$

La conclusion suit.

TOME $130-2002-\mathrm{N}^{\mathrm{O}} 3$ 


\section{Preuves des corollaires}

La preuve des corollaires 1.2 et 1.5 reprend la démarche de M. Waldschmidt dans la preuve du théorème 7.3.4 de [12], formalisée par J.-C. Moreau au paragraphe $1 \mathrm{~b}$ de [7] et reprise par la proposition 3.1 de [10]. Dans le cas complexe, le corollaire 1.5 découle immédiatement de cette dernière proposition appliquée au théorème 1.4. Pour la commodité du lecteur, on redonne ci-dessous l'argument dans le cas ultramétrique.

Preuve du corollaire 1.2. - On écrit $f=P+g$ où $P$ est un polynôme de degré total $<M$ et où $g$ possède un zéro d'ordre $\geq M$ à l'origine. On obtient alors

$|f|_{(r, \ldots, r)} \leq \max \left\{|P|_{(r, \ldots, r)},|g|_{(r, \ldots, r)}\right\} \leq \max \left\{\left(\frac{r}{\rho_{1}}\right)^{M}|P|_{\rho},\left(\frac{r}{R}\right)^{M}|g|_{(R, \ldots, R)}\right\}$.

Pour tout $(\omega, t) \in \Omega \times \mathcal{T}$, on trouve aussi $\left|P^{[t]}(\omega)\right| \leq \max \left\{\left|f^{[t]}(\omega)\right|,\left|g^{[t]}(\omega)\right|\right\}$ puisque $P=f-g$, et les inégalités de Cauchy livrent $\left|g^{[t]}(\omega)\right| \delta(E)^{t} \leq|g|_{\rho} \leq$ $\left(\rho_{1} / R\right)^{M}|g|_{(R, \ldots, R)}$. En appliquant le théorème 1.1 à $P$, on en déduit

$$
|P|_{\rho} \leq C_{1} \max \left\{\max _{(\omega, t) \in \Omega \times \mathcal{T}}\left|f^{[t]}(\omega)\right| \delta(E)^{t},\left(\frac{\rho_{1}}{R}\right)^{M}|g|_{(R, \ldots, R)}\right\} .
$$

La conclusion suit en majorant $|g|_{(R, \ldots, R)}$ par $|f|_{(R, \ldots, R)}$ et en observant que $C_{1}$ est $\geq 1$.

La preuve du corollaire 1.3 utilise le lemme suivant.

LEMME 5.1. - Supposons $K$ ultramétrique et soit $F$ un sous-corps localement compact de $K$. Désignons par q la cardinalité du corps résiduel de $F$ et par $\lambda$ le générateur $>1$ de $\left|F^{\times}\right|$. Soient $\delta, \rho$ des éléments de $\left|F^{\times}\right|$avec $0<\delta<\rho$ et soit $\mathcal{E}$ un sous-ensemble maximal de $F \cap B(0, \rho)$ tel que $\delta(\mathcal{E})>\delta$. Alors la cardinalité de $\mathcal{E}$ est $S=(\rho / \delta)^{\log _{\lambda} q}$, et on a

$$
\frac{\rho^{S}}{\Delta(\mathcal{E}) \delta(\mathcal{E})} \leq \lambda^{S /(q-1)}
$$

Démonstration. - Écrivons $\rho=\delta \lambda^{\nu}$. Alors $F \cap B(0, \rho)$ est la réunion de $q^{\nu}$ translatés de $F \cap B(0, \delta)$, et $\mathcal{E}$ consiste d'un point pris dans chacun de ceux-ci, donc $S=q^{\nu}$. Pour tout $a \in \mathcal{E}$ et pour $j=0, \ldots, \nu$, on trouve exactement $q^{j}$ points de $\mathcal{E}$ dans $B\left(a, \delta \lambda^{j}\right)$, donc

$$
\delta(\mathcal{E}) \Delta(\mathcal{E})=\delta \lambda \prod_{j=1}^{\nu}\left(\delta \lambda^{j}\right)^{q^{j}-q^{j-1}}=\delta^{q^{\nu}} \lambda^{\nu q^{\nu}-\left(q^{\nu}-q\right) /(q-1)} \geq \rho^{S} \lambda^{-S /(q-1)} .
$$

Preuve du corollaire 1.3. - Pour $i=1, \ldots, n$, on choisit un sous-ensemble maximal $E_{i}$ de $F \cap B\left(0, \rho_{i}\right)$ tel que $\delta\left(E_{i}\right)>\delta_{i}$, on note $S_{i}$ sa cardinalité, et on pose $E=E_{1} \times \cdots \times E_{n}$. Alors, pour chaque $\alpha \in E$, l'ensemble $\Omega$ contient 
au moins un point qui vérifie la condition (1.1) du théorème 1.1. Ce théorème s'applique donc. La conclusion suit en observant que, selon le lemme 5.1, on a $M_{i}=S_{i} T_{i}=L_{i}$ pour $i=1, \ldots, n$ et $\rho^{L} \Delta(E)^{-T} \delta(E)^{-T} \leq \lambda^{M /(q-1)}$.

Preuve du corollaire 1.6. - Pour $i=1, \ldots, n$, on désigne par $N_{i}$ la partie entière de $\rho_{i} /\left(60 \delta_{i}\right)$ et on pose

$$
E_{i}=F \cap\left\{\frac{\rho_{i}}{2 N_{i}}(a+b \sqrt{-1}) ; a, b \in \mathbb{Z},|a|,|b| \leq N_{i}\right\} .
$$

Alors on a $N_{i} \geq 4$ et $E_{i}$ est de cardinalité $S_{i}=\left(2 N_{i}+1\right)^{d}$, donc $M_{i} \leq L_{i}=$ $S_{i} T_{i} \leq 3 M_{i}$. De plus, pour tout $\alpha \in E_{i}$, on a $\delta\left(\alpha, E_{i}\right)=\rho_{i} /\left(2 N_{i}\right)$, et par suite

$$
|\alpha|+2 \delta\left(\alpha, E_{i}\right) \leq \rho_{i}\left(\frac{1}{\sqrt{2}}+\frac{1}{4}\right) \leq \rho_{i} .
$$

Donc, l'ensemble $E=E_{1} \times \cdots \times E_{n}$ vérifie la condition $B\left(\alpha, 2 \delta_{\alpha}\right) \subseteq B(0, \rho)$ pour tout $\alpha \in E$. Enfin, pour $i=1, \ldots, n$, on a $\delta\left(E_{i}\right) / 30=\rho_{i} /\left(60 N_{i}\right) \geq \delta_{i}$. Donc l'ensemble $\Omega$ rencontre le polydisque $B(\alpha, \delta(E) / 30)$ pour chaque $\alpha \in E$. Ainsi, les hypothèses du théorème 1.4 sont vérifiées. Donc, pour tout polynôme $P \in \mathbb{C}[x]$ de degré partiel $<M_{i} / m$ en $x_{i}$ pour $i=1, \ldots, n$, on a

$$
|P|_{\rho} \leq 2^{m|T|} \frac{(8 \rho)^{L}}{\Delta(E)^{T} \delta(E)^{T}} \max _{(\omega, t) \in \Omega \times \mathcal{T}}\left|P^{[t]}(\omega)\right| \delta(E)^{t} .
$$

Comme $|L| \leq 3 M$, il suffit pour conclure de montrer que, pour $i=1, \ldots, n$, on a $\delta\left(E_{i}\right) \leq 40 \delta_{i}$ et $\Delta\left(E_{i}\right) \delta\left(E_{i}\right) \geq\left(2^{-5} \rho_{i}\right)^{S_{i}}$. La première inégalité est immédiate. Pour démontrer la seconde, on peut supposer $\rho_{i}=1$. Alors, on a $\delta\left(E_{i}\right)=$ $1 /\left(2 N_{i}\right)>\mathrm{e}^{-N_{i}}$ et le lemme 5.2 de $[10]$ donne $\Delta\left(E_{i}\right) \geq\left(8 \mathrm{e}^{3}\right)^{-S_{i} / 2}$ (on applique ce lemme avec $N=N_{i}, x=1 /(2 N)$, et $\Sigma=\{x\}$ si $F=\mathbb{R}$ et $\Sigma=\{x, i x\}$ si $F=\mathbb{C})$. Cela donne bien $\Delta\left(E_{i}\right) \delta\left(E_{i}\right) \geq\left(8 \mathrm{e}^{4}\right)^{-S_{i} / 2} \geq 2^{-5 S_{i}}$.

\section{Compléments dans le cas ultramétrique}

Supposons $K$ ultramétrique et soit $\mathcal{O}=\{z \in K ;|z| \leq 1\}$ son anneau de valuation. Rappelons que, pour un polynôme $P \in K\left[x_{1}, \ldots, x_{n}\right]$, la norme $|P|_{(1, \ldots, 1)}=\max \left\{|P(z)| ; z \in \mathcal{O}^{n}\right\}$ est simplement le maximum des valeurs absolues de ses coefficients, c'est-à-dire sa hauteur. On la note $H(P)$. La proposition suivante justifie les remarques faites à la suite du corollaire 1.2 en montrant que la constante $C_{2}$ qui y apparaît ne peut être choisie indépendamment de l'ensemble $E$.

Proposition 6.1. - Supposons $n \geq 2$. Soient $E_{1}, \ldots, E_{n}$ des sous-ensembles finis de $\mathcal{O}$ de même cardinalité $S=S_{1}=\cdots=S_{n} \geq 2$. On suppose que le corps $K$ est algébriquement clos et que $C$ est une constante qui possède la propriété suivante. Pour tout polynôme $f \in K\left[x_{1}, \ldots, x_{n}\right]$ dont l'ensemble des

TOME $130-2002-\mathrm{N}^{\mathrm{O}} 3$ 
zéros dans $K^{n}$ contient une perturbation de $E=E_{1} \times \cdots \times E_{n}$ au sens restreint de Robba, et pour tout nombre réel $R \geq 1$, on a

$$
H(f) \leq C R^{-S}|f|_{(R, \ldots, R)} .
$$

Alors

$$
C \geq \max \left\{\frac{\delta \Delta\left(E_{i}\right)}{\Delta\left(E_{j}\right)} ; 1 \leq i, j \leq n\right\}
$$

où $\delta=\min \left\{\delta\left(E_{i}\right) ; 1 \leq i \leq n\right\}$.

Rappelons que la notion de perturbation d'ensemble produit est définie à la suite du théorème 1.1 .

Démonstration. - Pour des raisons de symétrie, il suffit de montrer que l'on a $C \geq \delta \Delta\left(E_{2}\right) / \Delta\left(E_{1}\right)$. Pour ce faire, on choisit un point $\alpha_{1}$ de $E_{1}$ tel que $\Delta\left(\alpha_{1}, E_{1}\right)=\Delta\left(E_{1}\right)$ et on pose

$$
\varphi\left(x_{1}\right)=\prod_{a \in E_{1} \backslash \alpha_{1}}\left(x_{1}-a\right)
$$

de sorte que $\left|\varphi\left(\alpha_{1}\right)\right|=\Delta\left(E_{1}\right)$. On choisit aussi un élément $b$ de $K$ avec $0<|b|<\delta \Delta\left(E_{2}\right)$. On pose encore

$$
\psi\left(x_{2}\right)=\prod_{a \in E_{2}}\left(x_{2}-a\right) \text { et } f\left(x_{1}, \ldots, x_{n}\right)=\varphi\left(\alpha_{1}\right) \psi\left(x_{2}\right)+b \varphi\left(x_{1}\right) .
$$

Soit $\Omega_{2}$ l'ensemble des racines dans $K$ du polynôme $\psi\left(x_{2}\right)+b$. Par construction, $f$ s'annule en chaque point de l'ensemble

$$
\Omega=\left(\left(E_{1} \backslash\left\{\alpha_{1}\right\}\right) \times E_{2} \cup\left\{\alpha_{1}\right\} \times \Omega_{2}\right) \times E_{3} \times \cdots \times E_{n} .
$$

Fixons temporairement $\alpha \in E_{2}$, et choisissons un point $\omega$ de $\Omega_{2}$ pour lequel $|\omega-\alpha|$ soit minimal. Comme $K$ est algébriquement clos, on peut écrire $\psi(x)+b=\left(x-\omega_{1}\right) \cdots\left(x-\omega_{S}\right)$, où $\omega_{1}, \ldots, \omega_{S}$ sont les éléments de $\Omega_{2}$ répétés un nombre de fois égal à leur multiplicité. La valeur absolue de la dérivée logarithmique de $\psi(x)+b$ au point $\alpha$ est

$$
\left|\frac{\psi^{\prime}(\alpha)}{\psi(\alpha)+b}\right|=\left|\sum_{i=1}^{S} \frac{1}{\alpha-\omega_{i}}\right| \leq \max _{1 \leq i \leq S} \frac{1}{\left|\alpha-\omega_{i}\right|}=\frac{1}{|\alpha-\omega|} .
$$

Comme $\left|\psi^{\prime}(\alpha)\right|=\Delta\left(\alpha, E_{2}\right) \geq \Delta\left(E_{2}\right)$ et que $|\psi(\alpha)+b|=|b|<\delta \Delta\left(E_{2}\right)$, cela implique $|\omega-\alpha|<\delta$. Cette observation montre que $\Omega$ contient une perturbation de $E$ au sens de Robba.

Ainsi $f$ vérifie les hypothèses de la proposition. Par conséquent, on doit avoir $H(f) \leq C R^{-S}|f|_{(R, \ldots, R)}$ pour tout nombre réel $R \geq 1$. Pour $R$ tendant vers l'infini, le membre de droite de cette inégalité tend vers $C\left|\varphi\left(\alpha_{1}\right)\right|=C \Delta\left(E_{1}\right)$. Comme $H(f) \geq|b|$, on en déduit $C \geq|b| \Delta\left(E_{1}\right)^{-1}$. La conclusion suit puisqu'on peut supposer $|b|$ arbitrairement voisin de $\delta \Delta\left(E_{2}\right)$.

BULletin DE LA SOCiÉtÉ MATHÉmATIQUE DE FRANCE 
On se propose maintenant de raffiner le corollaire 1.2 dans le cas où $r=$ $\rho_{i}=T_{i}=1$ (et donc $L_{i}=S_{i}$ ) pour $i=1, \ldots, n$. On commence par généraliser le théorème 1.1 sous les mêmes contraintes.

Proposition 6.2. - Soient $E_{1}, \ldots, E_{n}$ des sous-ensembles finis de $\mathcal{O}$ de cardinalités respectives $S_{1}, \ldots, S_{n} \geq 2$, et soit $\Omega$ une perturbation de $E=E_{1} \times$ $\cdots \times E_{n}$ au sens général indiqué à la suite du théorème 1.1 . On pose

$$
C=\prod_{i=1}^{n} \frac{1}{\Delta\left(E_{i}\right) \delta\left(E_{i}\right)} \quad \text { et } \quad C_{i}=C \max _{a \in E_{i}} \Delta\left(a, E_{i}\right) \delta\left(a, E_{i}\right) \quad \text { pour } i=1, \ldots, n \text {. }
$$

Alors, pour tout sous-ensemble $I$ de $\{1, \ldots, n\}$ et tout polynôme $P \in$ $K\left[x_{1}, \ldots, x_{n}\right]$ vérifiant $\operatorname{deg}_{x_{i}} P<S_{i}$ pour $i \in\{1, \ldots, n\} \backslash I$, on $a$

$$
H(P) \leq \max \left\{C \max _{\omega \in \Omega}|P(\omega)|, \max _{i \in I} C_{i} H\left(P_{i}\right)\right\}
$$

où, pour $i=1, \ldots, n$, on désigne par $P_{i}$ la somme des monômes de $P$ divisibles $\operatorname{par} x_{i}^{S_{i}}$.

Démonstration. - Pour des raisons d'homogénéité, on peut se restreindre aux polynômes $P$ de hauteur 1 . On démontre la proposition par récurrence sur la cardinalité de $I$. Si $I=\varnothing$, elle découle immédiatement du théorème 1.1. Fixons un sous-ensemble non vide $I$ de $\{1, \ldots, n\}$ et supposons la proposition vérifiée pour tous les sous-ensembles de $I$ distincts de $I$. Soit $k$ un élément de $I$ pour lequel $C_{k}$ est maximal et soit $P$ un polynôme de hauteur 1 vérifiant les conditions de la proposition pour l'ensemble $I$ choisi. On peut supposer $H\left(P_{k}\right)<1 / C_{k}$ sinon la proposition est trivialement vérifiée pour $P$. Posons $J=I \backslash\{k\}$. En divisant $P_{k}$ par le polynôme unitaire de degré $S_{k}$

$$
\psi=\prod_{a \in E_{k}}\left(x_{k}-a\right) \in \mathcal{O}\left[x_{k}\right],
$$

on obtient $P_{k}=A+\psi B$ où $A$ et $B$ sont des polynômes satisfaisant $\operatorname{deg}_{x_{i}} A<S_{i}$ pour $i \in\{1, \ldots, n\} \backslash J$ et $H(B) \leq H\left(P_{k}\right)$. On en déduit

$$
P=Q+\psi B
$$

où $Q=\left(P-P_{k}\right)+A$ satisfait aussi $\operatorname{deg}_{x_{i}} Q<S_{i}$ pour $i \in\{1, \ldots, n\} \backslash J$. Par hypothèse, la proposition est donc vérifiée pour l'ensemble $J$ et le polynôme $Q$. Puisque $H(P)=1$ et que $H(B)<1 / C_{k} \leq 1$, on a $H(Q)=1$. De plus, pour tout $i \in J$, on a $P_{i}=Q_{i}+\psi B_{i}$ où $Q_{i}$ (resp. $B_{i}$ ) désigne la somme des monômes de $Q$ (resp. $B$ ) divisibles par $x_{i}^{S_{i}}$. On en déduit

$$
H\left(P_{i}-Q_{i}\right) \leq H(B)<\frac{1}{C_{k}} \leq \frac{1}{C_{i}} .
$$

Donc, s'il existe un indice $i \in J$ tel que $H\left(Q_{i}\right) \geq 1 / C_{i}$, cela implique $H\left(P_{i}\right) \geq 1 / C_{i}$ pour ce même indice et la proposition est vérifiée pour notre 
choix de $I$ et de $P$. Sinon, il existe $\omega=\left(\omega_{1}, \ldots, \omega_{n}\right) \in \Omega$ avec $|Q(\omega)| \geq 1 / C$. Soit $a$ le point de $E_{k}$ pour lequel $\left|\omega_{k}-a\right|<\delta\left(a, E_{k}\right)$. On trouve

$$
|P(\omega)-Q(\omega)| \leq\left|\psi\left(\omega_{k}\right)\right| H(B)=\left|\omega_{k}-a\right| \Delta\left(a, E_{k}\right) H(B)<\frac{1}{C},
$$

donc $|P(\omega)| \geq 1 / C$ et la proposition est encore vérifiée pour $I$ et $P$. Par récurrence, elle est vraie pour tout choix de $I$.

Corollaire 6.3. - Soient $R=\left(R_{1}, \ldots, R_{n}\right)$ un $n$-uplet de nombres réels $\geq 1$ et $f: B(0, R) \rightarrow K$ une fonction analytique. Sous les hypothèses de la proposition 6.2 , on a

$$
|f|_{(1, \ldots, 1)} \leq \max \left\{C \max _{\omega \in \Omega}|f(\omega)|, \max _{1 \leq i \leq n} C_{i} R_{i}^{-S_{i}}|f|_{\left(1, \ldots, R_{i}, \ldots, 1\right)}\right\}
$$

où $\left(1, \ldots, R_{i}, \ldots, 1\right)$ désigne le $n$-uplet dont le $i$-ième élément est égal à $R_{i}$ et tous les autres égaux à 1 .

Démonstration. - Lorsque $f=P$ est un polynôme, cela découle immédiatement de la proposition car alors on a $H\left(P_{i}\right) \leq R_{i}^{-S_{i}}|P|_{\left(1, \ldots, R_{i}, \ldots, 1\right)}$ pour $i=1, \ldots, n$. Le cas général s'ensuit par continuité.

On conclut en montrant que les constantes $C_{1}, \ldots, C_{n}$ de la proposition 6.2 et de son corollaire 6.3 sont essentiellement optimales.

Proposition 6.4. - Soient $E_{1}, \ldots, E_{n}$ des sous-ensembles finis de $\mathcal{O}$ de cardinalités respectives $S_{1}, \ldots, S_{n} \geq 2$. Supposons que des constantes $C_{1}, \ldots, C_{n}$ possèdent la propriété suivante. Pour tout polynôme $f \in K\left[x_{1}, \ldots, x_{n}\right]$ dont l'ensemble des zéros dans $K^{n}$ contient une perturbation de $E=E_{1} \times \cdots \times E_{n}$ et pour tout choix de nombres réels $R_{1}, \ldots, R_{n} \geq 1$, on a

$$
H(f) \leq \max _{1 \leq i \leq n} C_{i} R_{i}^{-S_{i}}|f|_{\left(1, \ldots, R_{i}, \ldots, 1\right)} .
$$

Alors, pour $i=1, \ldots, n$, ces constantes vérifient

$$
C_{i} \geq \frac{\Delta\left(E_{i}\right) \max \left\{\delta\left(a, E_{i}\right) ; a \in E_{i}\right\}}{\Delta\left(E_{1}\right) \cdots \Delta\left(E_{n}\right)} .
$$

Démonstration. - Par symétrie, il suffit de minorer $C_{1}$. Pour cela, on choisit un point $\alpha_{1} \in E_{1}$ pour lequel $\delta\left(\alpha_{1}, E_{1}\right)$ est maximal et un élément quelconque $\omega_{1}$ de $K$ tel que $\left|\omega_{1}-\alpha_{1}\right|<\delta\left(\alpha_{1}, E_{1}\right)$. Pour $i=2, \ldots, n$, on choisit aussi un point $\alpha_{i} \in E_{i}$ tel que $\Delta\left(\alpha_{i}, E_{i}\right)=\Delta\left(E_{i}\right)$. On pose

$$
\varphi_{i}\left(x_{i}\right)=\prod_{a \in E_{i} \backslash \alpha_{i}}\left(x_{i}-a\right) \quad \text { pour } i=1, \ldots, n
$$

et

$$
f\left(x_{1}, \ldots, x_{n}\right)=\left(x_{1}-\alpha_{1}\right) \varphi_{1}\left(x_{1}\right) \prod_{i=2}^{n} \varphi_{i}\left(\alpha_{i}\right)+\left(\alpha_{1}-\omega_{1}\right) \prod_{i=1}^{n} \varphi_{i}\left(x_{i}\right) .
$$

BULletin DE LA SOCIÉtÉ MATHÉMATIQUE DE FRANCE 
Alors $f$ s'annule au point $\omega=\left(\omega_{1}, \alpha_{2}, \ldots, \alpha_{n}\right)$ et en chaque point de $E$ différent de $\alpha=\left(\alpha_{1}, \ldots, \alpha_{n}\right)$. Comme $\Omega=(E \backslash\{\alpha\}) \cap\{\omega\}$ est une perturbation de $E$, on en déduit que $f$ vérifie la condition (6.1) de la proposition pour tout choix de nombres réels $R_{1}, \ldots, R_{n} \geq 1$. Pour $R_{1}, \ldots, R_{n}$ tendant vers l'infini, le membre de droite de cette inégalité converge vers $C_{1} \prod_{i=2}^{n}\left|\varphi_{i}\left(\alpha_{i}\right)\right|=C_{1} \prod_{i=2}^{n} \Delta\left(E_{i}\right)$. Comme $H(f) \geq\left|\alpha_{1}-\omega_{1}\right|$, on en déduit

$$
C_{1} \geq \frac{\left|\alpha_{1}-\omega_{1}\right|}{\Delta\left(E_{2}\right) \cdots \Delta\left(E_{n}\right)}
$$

et la conclusion suit du fait que, pour un choix approprié de $\omega_{1}$, la quantité $\left|\alpha_{1}-\omega_{1}\right|$ peut être rendue arbitrairement voisine de $\delta\left(\alpha_{1}, E_{1}\right)$.

\section{BIBLIOGRAPHIE}

[1] Bombieri (E.) \& LANG (S.) - Analytic subgroups of group varieties, Invent. Math., t. 11 (1970), pp. 1-14.

[2] Grinspan (P.) - Measures of simultaneous approximation for quasiperiods of abelian varieties, J. Number Theory, t. 94 (2002), pp. 136-176.

[3] Masser (D.W.) - Elliptic functions and transcendence, Lecture Notes in Math., vol. 437, Springer-Verlag, Berlin, New York, 1975.

[4] _ Diophantine approximation and lattices with complex multiplication, Invent. Math., t. 45 (1978), pp. 61-82.

[5] , Polynomial interpolation in several complex variables, J. Approx. Theory, t. 24 (1978), pp. 18-34.

[6] Moreau (J.-C.) - Zéros de polynômes en plusieurs variables, C. R. Acad. Sci. Paris, Sér. A-B, t. 282 (1976), pp. A771-A774.

[7] _ Lemmes de Schwarz en plusieurs variables et applications arithmétiques, in Sém. P. Lelong, H. Skoda (Analyse) 1978/79, Lecture Notes in Math., vol. 822, Springer, Berlin, New York, 1980, pp. 174-190.

[8] Oesterlé (J.) - Réduction modulo $p^{n}$ des sous-ensembles analytiques fermés de $\mathbb{Z}_{p}^{N}$, Invent. Math., t. 66 (1982), pp. 325-341.

[9] RobBa (P.) - Lemmes de Schwarz et lemmes d'approximations p-adiques en plusieurs variables, Invent. Math., t. 48 (1978), pp. 245-277.

[10] RoY (D.) - Interpolation formulas and auxiliary functions, J. Number Theory, t. 94 (2002), pp. 248-285.

[11] Serre (J.-P.) - Dépendance d'exponentielles p-adiques, in Séminaire Delange-Pisot-Poitou, 1965-1966, exposé 15, 14 p.

[12] Waldschmidt (M.) - Nombres transcendants et groupes algébriques, Astérisque, vol. 69-70, Soc. Math. France., 1979, avec deux appendices par D. Bertrand et J.-P. Serre. 